\title{
Os modelos ortodônticos digitais são confiáveis? Uma revisão sistemática
}

(2) Matheus Melo Pithon ${ }^{1,3}$, Letícia Iandeyara Dantas de Andrade Sant'Anna ${ }^{1}$,

Tarcila Santana Matos ${ }^{1}$, Ana Carolina Dias Viana de Andrade ${ }^{2}$

Marcela Baraúna Magno3, Camila Silva de Amorim³, Orlando Motohiro Tanaka ${ }^{3}$, Lucianne Cople Maia ${ }^{3}$

${ }^{1}$ Departamento de Saúde I, Universidade Estadual do Sudoeste da Bahia - UESB

${ }^{2}$ Departamento de Saúde II, Universidade Estadual do Sudoeste da Bahia - UESB

${ }^{3}$ Departamento de Odontopediatria e Ortodontia da Universidade Federal do Rio de Janeiro - UFRJ

\section{$\square$ Matheus Pithon}

Av. Otávio Santos, 395, sala 705 Recreio

CEP: $45020-750$

Vitória da Conquista - BA

? matheuspithon@gmail.com

\section{RESUMO}

Introdução: Os modelos ortodônticos digitais são alternativas ao uso de modelos ortodônticos em gesso. Devido sua importância no diagnóstico, faz-se necessário averiguar a precisão dos softwares de modelos ortodônticos digitais disponíveis no mercado. Objetivo: Evidenciar qual software de modelos ortodônticos digitais possui maior confiabilidade.

Material e métodos: Foi realizada busca sistemática nas seguintes bases de dados eletrônicas: Scopus, PubMed, Web of Science, Embase e Cochrane, Grey Literature e Clinical Trials, sem limitações quanto ao ano de publicação ou idioma. Foram incluídos estudos laboratoriais que utilizaram softwares destinados a geração de modelos ortodônticos digitais, comparando-os com modelos ortodônticos em gesso. Após seleção por título e resumo, os artigos potencialmente elegíveis foram lidos na íntegra. A qualidade metodológica e o risco de viés dos artigos incluídos foram avaliados de acordo com as descrições dos modelos, técnicas de medição e análises estatísticas. A qualidade metodológica foi classificada em alta, moderada ou baixa. Resultados: 13.333 artigos foram encontrados nas bases de dados utilizadas. Após a aplicação dos critérios de elegibilidade e remoção de duplicações, restaram apenas 41 artigos. 29 foram considerados de qualidade moderada e 12 de alta qualidade. A classificação quanto à confiabilidade variou de menos confiaveis, equivalentes e a mais confiaveis. Apresentando diferenças estatisticas em suas mensurações $(p<0,05)$; sem significância clínica. Conclusão: os modelos ortodônticos digitais apresentaram mensurações acuradas, reprodutíveis e confiáveis quando comparado ao método convencional utilizando modelos de gesso.

Palavras-chave: modelos dentários, imagem tridimensional, ortodontia

\section{ABSTRACT}

Introduction: Digital models are an alternative for the use of orthodontic models in plaster. Due to its importance in the orthodontic dignified, it is necessary to ascertain the accuracy of the software of digital orthodontic models available in the market. Objective: To show which software of digital orthodontic models has greater reliability. Material and methods: A systematic search was performed in the following electronic databases: Scopus, PubMed, Web of Science, Embase and Cochrane, Gray Literature and Clinical Trials, with no limitations on year of publication or language. We included laboratory studies using digital orthodontic models, comparing them with orthodontic models of plaster through the evaluation of reliability through measurement techniques. After selection by title and abstract, potentially eligible articles have been read in full. The methodological quality and risk of bias of the included articles were evaluated according to the descriptions of the models, measurement techniques and statistical analyzes. The methodological quality was classified as high, moderate or low. Results: 13,333 articles were found in the databases used. After applying the eligibility criteria and removing duplicates, only 41 articles remained. 29 were considered of moderate quality and 12 of high quality. The ranking of digital models for reliability ranged from less reliable, equivalent, and more reliable. Presenting statistical differences in their measurements $(p<0.05)$, without clinical significance. Conclusion: The measurements made in digital models can be as accurate, reproducible and reliable as the conventional method using plaster models;

Keywords: dental models, three-dimensional image, orthodontics 
INTRODUÇÃO

A análise de modelos apresenta-se como um importante elemento na Ortodontia desde a época do seu surgimento como especialidade. Radiografias e fotografias digitais estão se tornando norma em registros ortodônticos assim como mais recentemente tem acontecido com os modelos de estudo eletrônicos (RHEUDE et al., 2005). Os modelos digitais foram introduzidos em 1990 seguindo os avanços da tecnologia digital (JOFFE, 2004; MAH, 2007). As vantagens incluem o não requerimento de espaço extra de armazenamento, facilidade de busca e classificação dos dados, a possibilidade de análise acurada e um método simplificado de transferência de dados (KANG et al., 2011; IM et al., 2014). Sendo assim os modelos digitais surgem como alternativas aos modelos de gesso.

Em estudos prévios, foi avaliada sistematicamente a validade do uso de modelos digitais ao serem comparados com modelos de gesso (FLEMING et al., 2011), todavia nenhuma revisão sistematica enfatizou qual software possui maior precisão, tendo em vista, as inúmeras opções disponiveis no mercado. Dessa forma, objetivou-se com esse estudo, buscar evidências na literatura que elucidem se os modelos ortodônticos digitais são tão confiáveis quanto os modelos em gesso.

\section{MATERIAL E MÉTODOS}

A metodologia empregada nessa revisão sistemática foi baseada nas diretrizes PRISMA (www.prisma-statement. org).

\section{Estratégia de busca}

Para identificação de artigos relevantes, sem limitações de ano de publicação e idioma, foi realizada uma busca nas seguintes bases de dados eletrônicas: Pubmed, Cochrane, Scopus, Embase, Web of Science, Clinical Trials e na Greyliterature. A estratégia de busca foi adequada para cada base de dados. Foram utilizados os seguintes descritores/MeSHterms: dentistry, orthodontics, technology, diagnosis, planning techniques, dental models, dimensional measurement accuracy, efficienc, treatment e as palavras-chave: digital models, digital record storage, digital dental casts, digital measurement method, 3-dimensional(3D) digital models, plaster models, plaster dental casts, accuracy, reproducibility, validity, virtual models. Os detalhes da estratégia de busca são apresentados na Tabela 1.

\section{Critérios de elegibilidade dos artigos}

$\mathrm{Na}$ presente revisão sistemática foram incluídos estudos laboratoriais nos quais foram utilizados modelos ortodônticos digitais (P), onde foi realizada avaliação da confiabilidade através de técnicas de medição (I) comparando esse método de diagnóstico com modelos em gesso convencionais (C) a fim de ratificar sua contribuição na prática clínica (O). Para que os artigos fossem selecionados e incluídos nessa revisão a partir do título e resumos, os mesmos deveriam satisfazer os seguintes critérios de inclusão: ser estudo laboratorial e utilizar modelos ortodônticos digitais. Como critérios de exclusão relatos de caso, estudos clínicos, revisão de literatura, livros didáticos, cartas editoriais. A seleção inicial se deu a partir da leitura dos títulos e resumos dos artigos encontrados. Aqueles que não eram relacionados com o tema foram excluídos, assim como os que apresentavam algum critério de exclusão previamente estabelecido. Os artigos em que o título e o resumo não apresentavam informações suficientes foram baixados e analisados totalmente para que se pudesse decidir sobre a sua elegibilidade. Os que apresentavam título dentro do tema porém o resumo não era disponibilizado também foram obtidos e analisados na íntegra (aqueles indisponíveis a serem baixados, solicitouse junto aos autores que os enviasse por e-mail). Os artigos que apareceram em mais de uma base de dados de pesquisa foram considerados apenas uma vez. Dois pesquisadores (T.M. e M.R.) fizeram as seleções independentes e os resultados foram comparados, com a finalidade de evitar discrepâncias que pudessem ocorrer durante a coleta dos dados. Se fossem encontradas discrepâncias entre os dois avaliadores em relação à inclusão ou exclusão de algum estudo, um terceiro avaliador seria adicionado (M.M.P.) em uma reunião de consenso a fim de eliminar as discrepâncias.

As listas de referências dos artigos selecionados foram avaliadas para verificar a existência de algum estudo que não fora encontrado outrora pelas bases de dados pesquisadas.

Nos casos em que dados adicionais fossem necessários, foram realizados contatos via e-mail com os autores de correspondência dos estudos, a fim de sanar as dúvidas em relação aos critérios de elegibilidade. Para ser aceito nesta revisão, os artigos deveriam relatar a utilização de modelos ortodônticos digitais e modelos de gesso convencionais, comparando a precisão das informações registradas nos mesmos.

\section{Avaliação da qualidade metodológica e risco de viés}

Um procedimento de pontuação foi desenvolvido a fim de se avaliar qualitativamente a metodologia dos artigos incluídos nesta revisão sistemática. Uma lista com 12 itens de avaliação foi aplicada, a qual incluiu os critérios de elegibilidade dos modelos ortodônticos, tamanho da amostra, descrição da condição dos modelos ortodônticos, discriminação do instrumento de medida, descrição da manipulação dos modelos, especificidade dos pontos de referência, abordagem do tempo de mensuração, registro das medidas obtidas, comparação com o grupo controle, randomização declarada, ocultação de pesquisadores e apresentação da significância estatística. 
Tabela 1: Base de dados, métodos de pesquisa e número de artigos obtidos

\begin{tabular}{lcc}
\hline Base de dados & Métodos de pesquisa & Resultados \\
\hline & orthodontic technology AND (diagnosis OR planning techniques)) OR ((dentistry OR \\
orthodontics) AND (dental models OR digital models OR digital records storage OR \\
digital dental casts OR digital measurement method, OR 3D models OR digital models \\
OR digital technology OR virtual models) OR (orthodontic technology AND (diagnosis \\
OR planning techniques) AND (plaster models OR plaster dental casts OR dimensional \\
measurement accuracy OR reproducibility OR validity OR treatment)) OR (orthodontic \\
technology AND digital models AND (validity OR treatment OR efficiency))
\end{tabular}

orthodontic technology AND (diagnosis OR planning techniques)) OR ((dentistry OR orthodontics) AND (dental models OR digital models OR digital records storage OR digital dental casts OR digital measurement method, OR 3D models OR digital models Cochrane OR digital technology OR virtual models) OR (orthodontic technology AND (diagnosis OR planning techniques) AND (plaster models OR plaster dental casts OR dimensional measurement accuracy OR reproducibility OR validity OR treatment)) OR (orthodontic technology AND digital models AND (validity OR treatment OR efficiency))

orthodontic technology AND (diagnosis OR planning techniques)) OR ((dentistry OR
orthodontics) AND (dental models OR digital models OR digital records storage OR
digital dental casts OR digital measurement method, OR 3D models OR digital models
OR digital technology OR virtual models) OR (orthodontic technology AND (diagnosis
OR planning techniques) AND (plaster models OR plaster dental casts OR dimensional
measurement accuracy OR reproducibility OR validity OR treatment)) OR (orthodontic
technology AND digital models AND (validity OR treatment OR efficiency))
orthodontic technology AND (diagnosis OR planning techniques)) OR ((dentistry OR
orthodontics) AND (dental models OR digital models OR digital records storage OR
digital dental casts OR digital measurement method, OR 3D models OR digital models
OR digital technology OR virtual models) OR (orthodontic technology AND (diagnosis
OR planning techniques) AND (plaster models OR plaster dental casts OR dimensional
measurement accuracy OR reproducibility OR validity OR treatment)) OR (orthodontic
technology AND digital models AND (validity OR treatment OR efficiency))
orthodontic technology AND (diagnosis OR planning techniques)) OR ((dentistry OR
orthodontics) AND (dental models OR digital models OR digital records storage OR
digital dental casts OR digital measurement method, OR 3D models OR digital models
OR digital technology OR virtual models) OR (orthodontic technology AND (diagnosis
OR planning techniques) AND (plaster models OR plaster dental casts OR dimensional
measurement accuracy OR reproducibility OR validity OR treatment)) OR (orthodontic
technology AND digital models AND (validity OR treatment OR efficiency))

orthodontic technology AND (diagnosis OR planning techniques)) OR ((dentistry OR orthodontics) AND (dental models OR digital models OR digital records storage OR digital dental casts OR digital measurement method, OR 3D models OR digital models

Clinical Trials OR digital technology OR virtual models) OR (orthodontic technology AND (diagnosis OR planning techniques) AND (plaster models OR plaster dental casts OR dimensional measurement accuracy OR reproducibility OR validity OR treatment)) OR (orthodontic technology AND digital models AND (validity OR treatment OR efficiency))

orthodontic technology AND (diagnosis OR planning techniques)) OR ((dentistry OR
orthodontics) AND (dental models OR digital models OR digital records storage OR digital
dental casts OR digital measurement method, OR 3D models OR digital models OR digital
technology OR virtual models) OR (orthodontic technology AND (diagnosis OR planning
techniques) AND (plaster models OR plaster dental casts OR dimensional measurement
accuracy OR reproducibility OR validity OR treatment)) OR (orthodontic technology AND digital
models AND (validity OR treatment OR efficiency))


No que tange aos critérios de elegibilidade dos modelos ortodônticos, seria atribuído ao artigo 1 ponto, se estes critérios fossem declarados, e nenhuma pontuação se não o fossem. Referente ao tamanho da amostra, o artigo foi pontuado com 1 ponto caso apresentasse uma amostra $\geq 10$ modelos, com 0,5 ponto se a amostra fosse $\geq 5$ e $<10$ modelos e nenhuma pontuação se a amostra fosse < 5 modelos. Quanto a descrição da condição dos modelos ortodônticos, o estado de conservação da amostra foi considerado, se fosse especificado pelo artigo o mesmo era pontuado com 1 ponto, e caso fosse abordado sem clareza ou não fosse abordado pelo artigo, este não era pontuado. Em relação à especificidade do instrumento de medida, quando o artigo discriminasse o instrumento utilizado, seria atribuído ao mesmo 1 ponto, caso não o fosse, não se atribuía pontuação. Quanto ao critério descrição da manipulação dos modelos ortodônticos, o artigo deveria especificar a forma como as medidas foram realizadas para obter 1 ponto, na ausência dessa informação, o mesmo não era pontuado. No que tange à especificidade dos pontos de referência, quando o artigo estabelecesse os parâmetros utilizados para aferição das medidas, atribuía-se 1 ponto ao mesmo, na ausência dessa informação não se atribuía pontuação. $\mathrm{Na}$ abordagem do tempo de mensuração, o artigo recebia 1 ponto caso fosse identificado o tempo útil das medidas obtidas, e não seria pontuado caso não apresentasse tal informação. No que tange ao critério de registro das medidas obtidas, atribuía-se 1 ponto ao artigo quando o mesmo apontasse os valores registrados nas mensurações, e não recebia pontuação quando não informasse os valores resultantes das aferições. Quanto aos critérios de comparação com o grupo controle, randomização declarada, ocultação de pesquisadores e apresentação de significância estatística, os artigos obtinham a pontuação de 1 ponto para cada um desses itens caso fossem abordados, e não seriam pontuados caso não os apresentasse. A qualidade metodológica dos artigos foi mensurada da seguinte maneira: baixa qualidade $(\leq 5)$, qualidade moderada $(>5$ e $<10)$ e qualidade alta $(\geq 10)$ (Tabela 2).

Tabela 2: Procedimento de pontuação qualitativa

\begin{tabular}{ll}
\hline Critérios de avaliação & Pontuação máxima (12) \\
\hline A - Critérios de elegibilidade dos modelos & 1 \\
B - Tamanho da amostra & 1 \\
C - Descrição da condição dos modelos & 1 \\
ortodônticos & 1 \\
D- Discriminação do instrumento de medida & 1 \\
E - Descrição da manipulação dos modelos & 1 \\
F - Especificidade dos pontos de referência & 1 \\
G - Abordagem do tempo de mensuração & 1 \\
H - Registro das medidas obtidas & 1 \\
I - Comparação com o grupo controle & 1 \\
\hline J - Randomização declarada & 1 \\
K - Ocultação de pesquisadores & 1 \\
\hline L - Significância estatística & \\
\hline
\end{tabular}

\section{RESULTADOS}

Um total de 13.333 artigos foi encontrado durante a pesquisa nas bases de dados eletrônicas. Os artigos não relacionados ao tema foram excluídos deste estudo, o que foi feito mediante a leitura dos títulos e resumos. Assim, 63 artigos foram selecionados. Após a remoção das duplicatas e atendendo os critérios de elegibilidade dessa revisão, 44 artigos foram incluídos para avaliação do texto completo. A Figura 1 ilustra os resultados das buscas.

Os estudos foram classificados de acordo com a qualidade metodológica e pontuados conforme as descrições apresentadas na Tabela 3. Três estudos foram excluídos do processo de extração de dados, uma vez que a sua qualidade metodológica foi pontuada como baixa(Asquith; Mcintyre,
2012; Murugesan et al., 2012; Kim et al., 2014(a)). Apenas 41 artigos preencheram todos os critérios de seleção e tiveram uma qualidade metodológica de moderada a alta, permitindo que os mesmos fossem considerados nesta revisão sistemática. Dos artigos incluídos, 29 apresentaram qualidade moderada e 12 qualidade alta, como pode ser observado na tabela 2.

Em relação aos critérios para avaliação metodológica, apenas 4 estudos abordaram o tempo de mensuaração dos modelos, a ausência dessa informação impossibilita a comparação entre métodos para avaliar qual o mais rápido. O critério de elegibilidade é de suma importância para que haja padronização dos modelos ao serem comparados, 10 estudos não contemplaram esse critério. Três estudos não discriminaram o instrumento de medida utilizado, 


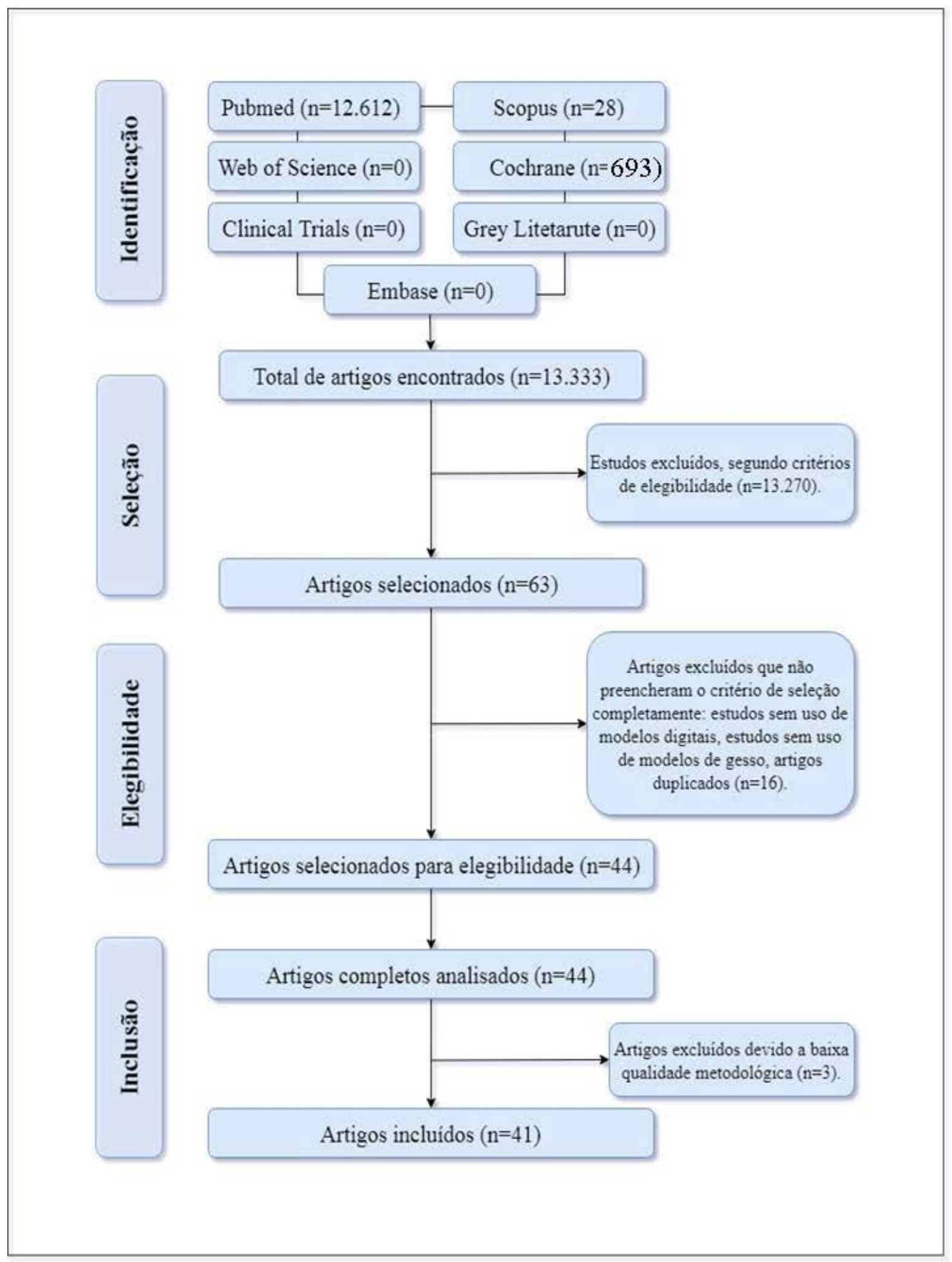

Figura 1: Fluxograma - Resultados das buscas nas bases de dados 
Tabela 3: Protocolo de pontuação qualitativa da metodologia dos artigos analisados

\begin{tabular}{|c|c|c|c|c|c|c|c|c|c|c|c|c|c|c|}
\hline Autor & $A$ & B & $\mathrm{C}$ & $\mathrm{D}$ & $E$ & $\mathrm{~F}$ & $\mathrm{G}$ & $\mathrm{H}$ & I & J & $\mathrm{K}$ & $\mathrm{L}$ & Total & Qualidade \\
\hline Abizadeh et al. (2012) & 1 & 1 & 1 & 1 & 1 & 1 & 0 & 1 & 1 & 1 & 1 & 1 & 11 & Alta \\
\hline Asquith; Mcintyre (2012) & 0 & 1 & 0 & 0 & 1 & 0 & 0 & 0 & 1 & 1 & 0 & 1 & 5 & Baixa* \\
\hline Bell et al. (2003) & 0 & 1 & 0 & 1 & 1 & 1 & 0 & 1 & 1 & 1 & 1 & 1 & 9 & Moderada \\
\hline Bootvong et al. (2010) & 1 & 1 & 0 & 1 & 0 & 1 & 0 & 1 & 1 & 1 & 1 & 1 & 9 & Moderada \\
\hline Camardella et al. (2017) & 1 & 1 & 0 & 1 & 1 & 1 & 0 & 1 & 1 & 1 & 0 & 1 & 9 & Moderada \\
\hline Costalos et al. (2005) & 1 & 1 & 0 & 0 & 0 & 1 & 0 & 1 & 0 & 0 & 1 & 1 & 6 & Moderada \\
\hline Czarnota et al. (2016) & 1 & 1 & 0 & 1 & 1 & 1 & 0 & 1 & 1 & 1 & 0 & 1 & 9 & Moderada \\
\hline Dalstra; Melsen (2009) & 0 & 0 & 0 & 1 & 0 & 1 & 0 & 1 & 1 & 1 & 1 & 1 & 7 & Moderada \\
\hline El-Zanaty et al. (2010) & 1 & 1 & 0 & 1 & 1 & 1 & 0 & 1 & 0 & 1 & 1 & 1 & 9 & Moderada \\
\hline Fu et al. (2017) & 1 & 1 & 1 & 1 & 1 & 1 & 0 & 1 & 1 & 1 & 1 & 1 & 11 & Alta \\
\hline Grewal et al. (2017) & 1 & 1 & 0 & 1 & 1 & 1 & 0 & 1 & 1 & 0 & 0 & 1 & 8 & Moderada \\
\hline Grunheid et al. (2014) & 0 & 1 & 0 & 1 & 1 & 1 & 1 & 1 & 1 & 1 & 1 & 1 & 10 & Alta \\
\hline Hazeveld et al. (2014) & 1 & 1 & 1 & 1 & 0 & 1 & 0 & 1 & 1 & 1 & 1 & 0 & 9 & Moderada \\
\hline Hildebrand et al. (2008) & 1 & 1 & 1 & 1 & 0 & 1 & 0 & 1 & 1 & 1 & 1 & 1 & 10 & Alta \\
\hline Im et al. (2014) & 1 & 1 & 0 & 1 & 1 & 1 & 0 & 1 & 1 & 0 & 1 & 1 & 9 & Moderada \\
\hline Jimenez-Gayosso et al. (2018) & 1 & 1 & 0 & 1 & 0 & 1 & 0 & 1 & 1 & 0 & 0 & 1 & 7 & Moderada \\
\hline Kasparova et al. (2013) & 0 & 1 & 0 & 1 & 0 & 1 & 0 & 1 & 1 & 1 & 1 & 1 & 8 & Moderada \\
\hline Keating et al. (2008) & 1 & 0,5 & 1 & 1 & 0 & 1 & 0 & 1 & 1 & 1 & 1 & 1 & 9,5 & Moderada \\
\hline Kim, J. H. et al. (2014) & 0 & 1 & 0 & 1 & 1 & 1 & 0 & 1 & 1 & 0 & 1 & 0 & 7 & Moderada \\
\hline Kim, S. Y. et al.(2014) & 0 & 1 & 0 & 0 & 0 & 1 & 0 & 0 & 1 & 0 & 1 & 0 & 4 & Baixa* \\
\hline Kim, J. et al. (2014) & 1 & 1 & 0 & 1 & 0 & 1 & 0 & 1 & 1 & 0 & 1 & 1 & 8 & Moderada \\
\hline Koretsi et al. (2018) & 1 & 1 & 0 & 1 & 1 & 1 & 0 & 1 & 1 & 1 & 1 & 1 & 11 & Alta \\
\hline Leifert et al., (2009) & 1 & 1 & 0 & 1 & 0 & 1 & 0 & 1 & 1 & 1 & 1 & 1 & 9 & Moderada \\
\hline Luu et al. (2014) & 1 & 1 & 0 & 1 & 0 & 0 & 1 & 0 & 1 & 1 & 1 & 1 & 8 & Moderada \\
\hline Mangiacapra et al. (2009) & 0 & 0,5 & 0 & 1 & 0 & 1 & 0 & 1 & 1 & 0 & 0 & 1 & 5,5 & Moderada \\
\hline Mullen et al. (2007) & 1 & 1 & 0 & 1 & 1 & 1 & 1 & 1 & 1 & 1 & 0 & 1 & 10 & Alta \\
\hline Murugesan et al. (2012) & 0 & 0 & 0 & 1 & 0 & 0 & 0 & 1 & 1 & 0 & 1 & 0 & 4 & Baixa* \\
\hline Nalcaci et al. (2013) & 1 & 1 & 1 & 1 & 0 & 1 & 0 & 1 & 1 & 0 & 1 & 1 & 9 & Moderada \\
\hline Okunami et al. (2007) & 1 & 1 & 0 & 1 & 0 & 1 & 0 & 1 & 1 & 0 & 1 & 1 & 8 & Moderada \\
\hline Rangel et al. (2013) & 1 & 1 & 1 & 1 & 0 & 0 & 0 & 1 & 1 & 1 & 1 & 1 & 9 & Moderada \\
\hline Rheude et al. (2005) & 1 & 1 & 0 & 0 & 0 & 0 & 0 & 0 & 1 & 1 & 1 & 1 & 6 & Moderada \\
\hline Rheude et al. (2005) & 1 & 1 & 1 & 1 & 1 & 1 & 0 & 1 & 1 & 1 & 1 & 1 & 11 & Alta \\
\hline Santoro et al., (2003) & 1 & 1 & 1 & 1 & 1 & 1 & 0 & 1 & 1 & 1 & 1 & 1 & 11 & Alta \\
\hline Sfondrini et al., (2018) & 1 & 1 & 0 & 1 & 1 & 1 & 0 & 1 & 1 & 1 & 0 & 1 & 10 & Alta \\
\hline Sjogren et al., (2010) & 1 & 1 & 0 & 1 & 1 & 1 & 0 & 1 & 0 & 0 & 1 & 1 & 8 & Moderada \\
\hline Stevens et al., (2006) & 1 & 1 & 1 & 1 & 1 & 1 & 0 & 1 & 1 & 1 & 1 & 1 & 11 & Alta \\
\hline Tavares et al., (2017) & 1 & 1 & 1 & 1 & 1 & 0 & 0 & 1 & 1 & 1 & 1 & 1 & 10 & Alta \\
\hline Veenema et al., (2009) & 1 & 1 & 1 & 0 & 0 & 0 & 0 & 1 & 1 & 1 & 1 & 1 & 8 & Moderada \\
\hline Watanabe-Kanno et al. (2009) & 1 & 1 & 0 & 1 & 1 & 1 & 0 & 1 & 1 & 1 & 1 & 1 & 10 & Alta \\
\hline Watanabe-Kanno et al. (2010) & 1 & 1 & 0 & 1 & 1 & 0 & 0 & 1 & 1 & 1 & 1 & 1 & 9 & Moderada \\
\hline Wiranto et al. (2013) & 1 & 1 & 0 & 1 & 1 & 1 & 0 & 1 & 1 & 0 & 1 & 1 & 9 & Moderada \\
\hline Yuan et al., (2013) & 1 & 1 & 0 & 1 & 0 & 1 & 1 & 1 & 1 & 0 & 0 & 1 & 8 & Moderada \\
\hline Zhang et al., (2016) & 1 & 1 & 0 & 1 & 1 & 1 & 0 & 1 & 1 & 0 & 0 & 1 & 8 & Moderada \\
\hline Zilberman et al. (2003) & 0 & 1 & 0 & 1 & 0 & 1 & 0 & 1 & 1 & 0 & 1 & 1 & 7 & Moderada \\
\hline
\end{tabular}

* artigos excluídos

A-Critérios de elegibilidade dos modelos; B-Tamanho da amostra; C-Descrição da condição dos modelos ortodônticos; D-Discriminação do instrumento de medida; E-Descrição da manipulação dos modelos; F-Especificidade dos pontos de referência; G-Abordagem do tempo de mensuração; H-Registro das medidas obtidas; I-Comparação com o grupo controle; J-Randomização declarada; K-Ocultação de pesquisadores; L-Significância estatística. 
prejudicando a capacidade de reprodutibilidade do método empregado, assim como 6 estudos não estabeleceram pontos de referências para as medidas.

No que concerne a amostra dos estudos incluídos, apenas 3 estudos apresentaram amostras insatisfatórias. O registro das medidas não foi detalhado em 2 estudos. Nove estudos não declaram realizar a ocultação dos pesquisadores, enquanto 14 estudos não relataram realizar randomização dos modelos analisados.

\section{Modelos de gesso versus modelos digitais}

No que tange à avaliação de modelos digitais foi identificada na presente revisão a resolução dos instrumentos de medidas variou de $0.1 \mathrm{~mm}$ a $0.001 \mathrm{~mm}$. A condição dos modelos de estudo relatada apresentou uma boa qualidade sem a presença de fraturas (SANTORO et al., 2003; HILDEBRAND et al., 2008; KEATING et al., 2008; Veenema et al., 2009; NALCACI et al., 2013; HAZEVELD et al., 2014), ranhuras (KEATING et al., 2008), bolhas(SANTORO et al., 2003; STEVENS et al., 2006; HILDEBRAND et al., 2008; KEATING et al., 2008; NALCACI et al., 2013; HAZEVELD et al., 2014) ou irregularidades (SANCHES et al., 2013) (Tabela 4). Na abordagem do tempo útil de mensuração o mesmo variou, com a aquisição dos dados sendo alcançada em menos de 20 minutos(YUAN et al., 2013), com a seguinte ordem de alcance: Emodels (2 minutos e 48 segundos), SureSmile ( 3 minutos e 12 segundos) e AnatoModels (4 minutos e 32 segundos) comparadas aos modelos de gesso (4 minutos e 29 segundos) (GRUNHEID et al., 2014). Um estudo relatou que o tempo para realização dos cálculos nos modelos de gesso foi em média de 65,6 $\pm 47,0$ segundos menor do que os Emodels ( $p$.0001) (MULLEN et al., 2007). Em relação aos AnatoModels o tempo para realização das mensurações foi considerado em média 3,96 minutos maior do que nos modelos de gesso, sendo a confiabilidade intraexaminador de $95 \%$ ( $p=0.05$ ) (LUU et al., 2014).

No que se refere a média dos valores relacionados a confabilidade intraexaminador a mesma foi superior a 0,8 (Kim, J. H. et al., 2014) e levemente maior para os modelos digitais $(p<.0001)$ (COSTALOS et al., 2005;
Kim, J. et al., 2014) ( $p$ > 0.75) (EL-ZANATY et al., 2010). Constatou-se ainda, que a diferença média entre as medidas diretas e computadorizadas foi de $0,04 \mathrm{~mm}$ ( $p$ $=0.05$ ) (YUAN et al., 2013) e que as medidas obtidas usando modelos digitais, foram no geral menores do que aquelas obtidas usando modelos de gesso ( $p<$ $0.001)$ (NALCACI et al., 2013), $(p<0.05)$ (WATANABEKANNO et al., 2009), $(p=0.0124)$ (Santoro et al., 2003). Houve diferenças estatisticamente signifcante entre os grupos, quanto à alinhamento, contatos oclusais e overjet $(p<.05)$ (HILDEBRAND et al., 2008) conforme mostrado na Tabela 5, essa diferença foi maior para avaliação do overjet.

Em relação a reprodutibilidade das medidas, após a repetição de 10 mensurações, constatou-se a mesma sendo melhor para os modelos digitais onde o desvio padrão não execedeu a $0,10 \mathrm{~mm}$ ao contrário dos modelos de gesso no qual o mesmo foi quase $0,40 \mathrm{~mm}$ ( $p$ < 0.01) (DALSTRA; MELSEN, 2009). O comprimento do arco foi $1.5 \pm 1.36$ maior nos modelos de gesso do que nos modelos digitais ( $p<.0001$ ) (MULLEN et al., 2007). As medidas da largura dos dentes foram similares entre os modelos Orthocad e de gesso, a diferença média foi menor que 0,3 mm ( $p>0.05)$, (BOOTVONG et al., 2010). As medidas realizadas com SureSmile foram seguidas pelas demais sendo os modelos digitais da AnatoModelds os menos acurados ( $p<0.05)$ (GRUNHEID et al., 2014). As diferenças nas mensurações entre modelos de gesso, DigiModels e Lava models não excederam 0,2 mm ( $p<$ 0.05) (WIRANTO et al., 2013). Opondo-se aos demais meios de mensuração de modelos digitias, as medidas no RepRap3D demonstraram ser as mesmas nos modelos de gesso com desvio padrão menor que $0,5 \mathrm{~mm}$ (KASPAROVA et al., 2013).

Observa-se que existe diferença estatística signifcativa entre as mensurações nos modelos digitais para os modelos de gesso ( $p<0.05$ )(CAMARDELLA et al., 2017; TAVARES et al., 2017), no entanto não a signifcância clínica, supõese que esta diferença ocorra devido a ténica de confecção do modelo digital(CZARNOTA et al., 2016), sendo assim esperado que alguns parâmetros de espaço occora devido ao erro cumulativo(KORETSI et al., 2018).

Tabela 4: Descrição dos estudos incluídos

\begin{tabular}{lccc}
\hline & & Amostra & Intervenção \\
\hline Autor & Total & Condição dos modelos & Instrumento de medida \\
\hline Abizadeh et al. (2012) & 1 & $\begin{array}{c}\text { Modelos com vazios, dentes fraturados ou algum } \\
\text { outro dando foram excluídos }\end{array}$ & Convencional: paquimetro digital \\
Bell et al. (2003) & 22 & & Convencional: paquimetro digital \\
Bootvong et al. (2010) & 60 & - & Convencional: paquimetro digital \\
& & & Digital: -
\end{tabular}


Tabela 4: Continuação

\begin{tabular}{|c|c|c|c|}
\hline Camardella et al. (2017) & 28 & - & $\begin{array}{l}\text { Convencional: paquimetro digital } \\
\text { Digital: TRIOS Color Scanner/ Ortho } \\
\text { Analyzer software }\end{array}$ \\
\hline Costalos et al. (2005) & 48 & - & - \\
\hline Czarnota et al. (2016) & 20 & - & $\begin{array}{c}\text { Convencional: paquimetro digital } \\
\text { Digital: D700 Scanner/Ortho Analyzer } \\
\text { software }\end{array}$ \\
\hline Dalstra; Melsen (2009) & 3 & - & $\begin{array}{c}\text { Convencional: paquimetro digital } \\
\text { Digital: - }\end{array}$ \\
\hline El-Zanaty et al. (2010) & 34 & - & $\begin{array}{l}\text { Convencional: paquimetro digital } \\
\text { Digital: Programa de medições } \\
\text { dentárias com base em } 3 \text { dimensões } \\
\text { (3DD) }\end{array}$ \\
\hline
\end{tabular}

Vazados em forma padrão, sem vazios e fraturas e uniformemente

Fu et al. (2017) polido, registro claramente a dentição completa, osso basal, giro da mucosa,

frênulo, rugas palatinas e outras estruturas anatômicas

\begin{tabular}{|c|c|c|c|}
\hline Grewal et al. (2016) & 30 & - & $\begin{array}{c}\text { Convencional: paquimetro digital } \\
\text { Digital: Ortho Insight Scanner / Cloud } \\
\text { Software }\end{array}$ \\
\hline Grunheid et al. (2014) & 30 & - & $\begin{array}{c}\text { Convencional: paquimetro digital } \\
\text { Digital: Emodel } \\
\text { SureSmile software } \\
\text { AnatoModels }\end{array}$ \\
\hline Hildebrand et al. (2008) & 36 & $\begin{array}{c}\text { Sem bolhas, sem dentes quebrados, } \\
\text { retificados }\end{array}$ & $\begin{array}{c}\text { Convencional: Medidor de medição ABO } \\
\text { Digital: } A B O \text { OGS software }\end{array}$ \\
\hline Im et al. (2014) & 10 & - & $\begin{array}{c}\text { Convencional: paquimetro digital } \\
\text { Digital: Modelo de prototipagem rápida } \\
\text { (Projet) }\end{array}$ \\
\hline $\begin{array}{l}\text { Jimenez-Gayosso et al. } \\
\text { (2018) }\end{array}$ & 30 & - & $\begin{array}{l}\text { Convencional: paquimetro digital } \\
\text { Digital: Maestro3D Ortho Studio scanner }\end{array}$ \\
\hline Kasparova et al. (2013) & 10 & - & $\begin{array}{c}\text { Convencional: paquimetro digital } \\
\text { Digital: - }\end{array}$ \\
\hline Keating et al. (2008) & 30 & $\begin{array}{l}\text { Sem marcas na superficie, sem perda } \\
\text { dentária, sem vazios, fraturas ou } \\
\text { degraus nos pontos de contato }\end{array}$ & $\begin{array}{l}\text { Convencional: paquimetro digital } \\
\text { Digital: Scaner a laser sem contato }\end{array}$ \\
\hline Kim, J. H. et al. (2014) & 60 & & $\begin{array}{l}\text { Convencional: paquimetro digital } \\
\text { Digital: Ortho Insight 3D laser scanner }\end{array}$ \\
\hline Kim, S. Y. et al.(2014)a & 20 & - & $\begin{array}{l}\text { Convencional: Não menciona } \\
\text { Digital: Scanner de luz branca sem } \\
\text { contato/Delcam Copycad }\end{array}$ \\
\hline Koretsi et al. (2017) & 48 & - & $\begin{array}{l}\text { Convencional: paquimetro convencional } \\
\text { Digital: OrthoX Scan/ Ivorißanalyze }\end{array}$ \\
\hline Leifert et al., (2009) & 50 & - & $\begin{array}{c}\text { Convencional: paquimetro ortodontico } \\
\text { digital } \\
\text { Digital: OrthoCAD }\end{array}$ \\
\hline
\end{tabular}


Tabela 4: Continuação

\begin{tabular}{|c|c|c|c|}
\hline Luu et al. (2014) & 30 & - & $\begin{array}{c}\text { Convencional: paquimetro digital } \\
\text { Digital: Anatomodels (Invivo software) }\end{array}$ \\
\hline Mangiacapra et al. (2009) & 5 & - & $\begin{array}{l}\text { Convencional: paquimetro digital } \\
\text { Digital: Ortho3D software }\end{array}$ \\
\hline Mullen et al. (2007) & 30 & - & $\begin{array}{l}\text { Convencional: paquimetro digital } \\
\text { Digital: Emodel software }\end{array}$ \\
\hline Nalcaci et al. (2013) & 20 & $\begin{array}{c}\text { Sem vazios, sem bolhas ou fraturas no } \\
\text { dentes. }\end{array}$ & $\begin{array}{c}\text { Convencional: paquimetro digital } \\
\text { Digital: Ortho Three-dimensional } \\
\text { Models (O3DM) }\end{array}$ \\
\hline Okunami et al. (2007) & 30 & - & $\begin{array}{c}\text { Convencional: Medidor de medição } \\
\text { Digital: OrthoCAD }\end{array}$ \\
\hline Rangel et al. (2013) & 10 & $\begin{array}{c}\text { Sem danos, presença de todos os dentes, } \\
\text { morfologia normal dos dentes, sem } \\
\text { atrição visivel, cáries ou restaurações. }\end{array}$ & $\begin{array}{l}\text { Convencional: Paquimetro digital } \\
\text { Digital: Software MaxilimH 2.3.0 }\end{array}$ \\
\hline Rheude et al. (2005) & 7 & - & - \\
\hline Sanches et al. (2013) & 10 & $\begin{array}{l}\text { Todos os dentes apresentando } \\
\text { morfologianormal, ausência de } \\
\text { irregularidades }\end{array}$ & $\begin{array}{l}\text { Convencional: paquimetro digital } \\
\text { Digital: Scanner Orthodontic 3D }\end{array}$ \\
\hline Santoro et al. (2003) & 20 & Sem vazios ou bolhas, sem fraturas & $\begin{array}{c}\text { Convencional: paquimetro digital } \\
\text { Digital: OrthoCAD }\end{array}$ \\
\hline Sfondrini et al., (2017) & 14 & - & $\begin{array}{c}\text { Convencional: Paquimetro analogico } \\
\text { Digital: Trios } 3 \text { Mono Intraoral } \\
\text { Scanner / Ortho Analyzer software }\end{array}$ \\
\hline Sjogren et al., (2010) & 20 & - & $\begin{array}{c}\text { Convencional: paquimetro digital } \\
\text { Digital: - }\end{array}$ \\
\hline Stevens et al., (2006) & 24 & Sem bolhas positivas ou negativas, & $\begin{array}{l}\text { Convencional: paquimetro digital } \\
\text { Digital: Emodel software }\end{array}$ \\
\hline Tavares et al., (2017) & 37 & $\begin{array}{c}\text { Perfeito estado de preparação e } \\
\text { conservação, sem bolhas positivas ou } \\
\text { negativas ou defeitos na coroa dentária. }\end{array}$ & $\begin{array}{l}\text { Convencional: paquimetro digital } \\
\text { Digital: Ortho Insight 3D }\end{array}$ \\
\hline Veenema et al., (2009) & 30 & $\begin{array}{l}\text { Sem caracteristicas que possam alterar } \\
\text { o diametro mesiodistal ou bucolingual, } \\
\text { como restaurações, cáries, atrições ou } \\
\text { fraturas }\end{array}$ & - \\
\hline Watanabe-Kanno et al. (2009) & 15 & - & $\begin{array}{l}\text { Convencional: paquimetro digital } \\
\text { Digital: Cécile3 software }\end{array}$ \\
\hline Watanabe-Kanno et al. (2010) & 15 & - & $\begin{array}{l}\text { Convencional: paquimetro digital } \\
\text { Digital: Cécile3 software }\end{array}$ \\
\hline Wiranto et al. (2013) & 22 & - & $\begin{array}{c}\text { Convencional: paquimetro digital } \\
\text { Digital: Digi Model software } \\
\text { Lava Model software }\end{array}$ \\
\hline Yuan et al., (2013) & 10 & - & $\begin{array}{l}\text { Convencional: paquimetro digital } \\
\text { Digital: Software SPSS } 19.0\end{array}$ \\
\hline Zhang et al., (2017) & 20 & - & $\begin{array}{l}\text { Convencional: paquimetro digital } \\
\text { Digital: iTero Scanner }\end{array}$ \\
\hline Zilberman et al. (2003) & 20 & - & $\begin{array}{c}\text { Convencional: paquimetro digital } \\
\text { Digital: OrthoCAD }\end{array}$ \\
\hline
\end{tabular}


Tabela 5: Resumo dos resultados

\begin{tabular}{lll}
\hline Autor & $\begin{array}{c}\text { Tempo de } \\
\text { mensuração }\end{array}$ Registro das medidas obtidas \\
\hline
\end{tabular}

Abizadeh et al. (2012)

As diferenças estatisticamente significativas foram para largura intercanina superior $(0,14 \mathrm{~mm})$ e para a altura vertical inferior $(1,58 \mathrm{~mm})(\mathrm{p}<0,05)$.

Bell et al. (2003)

A média das diferenças de mensurações entre os modelos convencionais para os modelos digitais foi de 0.16 e $0.38 \mathrm{~mm}(p<0.05)$.

Bootvong et al. (2010)

A mensuração da discrepância entre as linhas intercaninos, intermolares, overjet, overbite, linha média, análise espacial e largura dos dentes apresentaram magnitudes similares (ICC $>0,7$ ).

Camardella et al. (2017)

$-$

Para vários parâmetros, foi encontrado diferença estatistica entre as medidas dos dois modelos. No entanto, a maioria dessas discrepâncias não foi considerada clinicamente significativa.

Costalos et al. (2005)

Os modelos de gessos convencionais possuem moderada confiabilidade, enquanto os modelos digitais possuem alta confiabilidade ( $p<0.0001)$.

Foram observadas diferenças estatisticas significativas em alguns

Czarnota et al. (2016) parametros como, deslocamento no segmento anterior de mandibular, overbite, distância intermolar na maxila, indice de Little e o indice de soma dos incisivos maxilares e mandibulares.

Dalstra; Melsen (2009)

O comprimento do arco e o overjet foram significativamente maiores quando medidos no modelo de gesso do que no modelo digital ( $p<$ 0,001).

As medidas mesiodistais na maxila para os segundos pré-molares direito e esquerdo, incisivo central esquerdo e primeiro molar direito, assim

El-Zanaty et al. (2010) - $\quad$ como as medidas mesiodistais na mandibula para os incisivos centrais direito e esquerdo, canino direito e primeiro pre-molar esquerdo tiveram concordância ( $p>0,75)$.

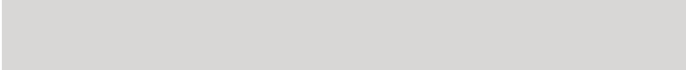

Fu et al. (2017)

As diferenças médias entre as medições dos modelos fotogramétricos 3D e os modelos de gesso variaram de 0,011 a 0,402 mm. As diferenças médias entre as medições obtidas pelos modelos fotogramétricos $3 \mathrm{D}$ e os modelos de gesso não foram significativos, exceto para o perímetro do arco inferior $(p>0.05)$, e todas as diferenças foram consideradas como normalmente aceitável $(<0,5 \mathrm{~mm})$.

Grewal et al. (2016)

Não foi encontrada diferença nas medições de análise do espaço total na arcada superior $(p=0.79)$ e inferior $(p=0.69)$.

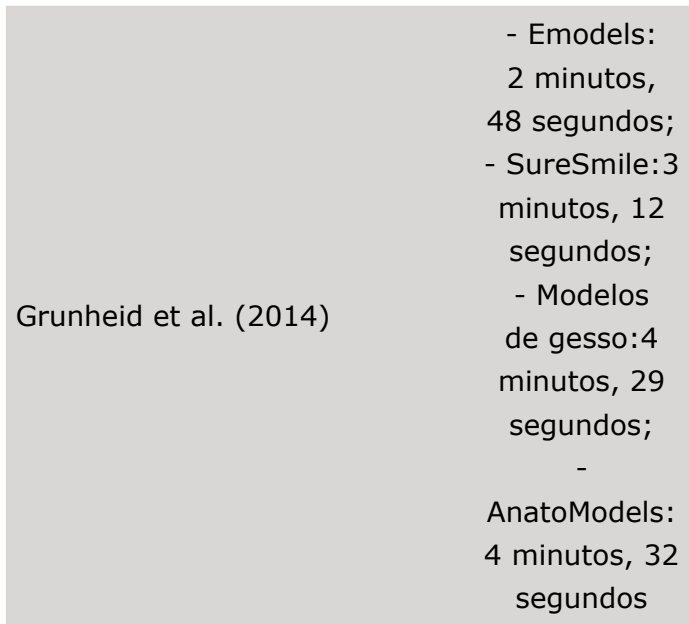

Hildebrand et al. (2008)
A precisão das medidas foi melhor no software s SureSmile, seguido por Emodels. As medições nos AnatoModels foram os menos precisas $(p<0.05)$.
Para o alinhamento, contato oclusal e overjet houve diferença estatistica significante $(p<0,05)$. 
Tabela 5: Continuação

Im et al. (2014)

Jimenez-Gayosso et al.

(2018)

Keating et al. (2008)

Kim, J. H. et al. (2014)

Kim, S. Y. et al.(2014)

Koretsi et al. (2017)

Leifert et al., (2009)
Diferenças estatisticas significantes foram observadas nas medidas de overjet e contato oclusal ( $p<0,01)$.

Nas medidas dos modelos do prétratamento foram observadas diferenças

na largura intermolar maxilar ( $\mathrm{p}=$

$0.030)$ e na altura do palto ( $p=0.002$.).

Já, as medidas do pos-tratamento apresentaram diferença entre os modelos para os seguintes parametros: largura intercanino mandibular ( $\mathrm{p}=$ $0.001)$, altura do palato ( $p=0.001)$, overjet $(p<0.006)$, overbite $(p=0.005)$, perímtro da maxila arco $(p=0.012)$, e no perímetro do arco mandibular $(p=0.028)$.

A precisão das medidas de distância nos modelos de gesso e nas cópias impressas

RepRap 3D foi a mesma $(p<0.05)$.

As mensurações nos planos $X$ e $Y$ não apresentaram diferença estatistica nos modelos analisados ( $p>0.5$ ). No entanto, no plano $Z$ as mensurações foram estatisticamente diferentes $(p<0,001)$.

A diferença média entre modelos de gesso e modelos PUT variaram de 0,07 $\mathrm{mm}$ a 0,33 $\mathrm{mm}$.

A diferença média entre os modelos digitais para os modelos de gesso e $\leq$ $0,2 \mathrm{~mm} 3 \pm 0,169 \mathrm{~mm}$. A largura do arco maxilar e do arco mandibular exibiram excelente concordância $(p<0,05)$.

$O$ viés entre os métodos foi estatisticamente significativo, mas menos de $0,5 \mathrm{~mm}$ para $87,2 \%$ dos resultados.

A diferença total maxila e mandíbula teve amplos limites de concordância, mas o viés entre os métodos foi menor que o viés entre intra-observador.

Arco superior: diferença de $0,4 \mathrm{~mm}$, sem

diferença estatistica significante Arco inferior: não houve diferenças 
Tabela 5: Continuação

- Modelo de gesso:

10 minutos

Luu et al. (2013)
- AnatoModel:

6 minutos
Comparando as medições dos modelos digitais com os modelos de gesso percebe-se pouca concordância com médias de diferenças baixas.

Houve diferença estatística nas médias das medições dos dentes $11,12,13,15,21,22,23$, 24,25 , largura intermolar inferior, perímetro do arco maxilar e mandibular, apinhamento, análise de Bolton, no entanto, apenas o perimetro do arco maxilar tinha um magnitude de diferença média, 3,38 mm e IC $95 \%(2,48 ; 4,28)$, que ultrapassaram o limiar clinicamente significativo

$$
(p<0,05) \text {. }
$$

Não houve diferenças estatisticas para as medições nos modelos digitais para os modelos convencionais nos seguintes parametros: distância canina ( $p=0.11)$, distância inter-molar $(p=0.24)$, comprimento distal primeiro molar inferior direito ( $p=0.4$ ), comprimento mesiodistal do incisivo lateral inferior direito $(p=0.39)$. No entanto, o erro de medição para overbite foi menor para modelos digitais (média

$=0,24$, DP $=0,21$ ) comparado aos modelos tradicionais (média $=0,69, \mathrm{DP}=0.53$ ) $(p<0.001)$.
Diferenças significativas foram encontradas entre o tempo

Mullen et al. (2007)

Nalcaci et al. (2013)

Okunami et al. (2007)

Rangel et al. (2013)

Rheude et al. (2005) para executar os cálculos nos modelos de gesso, média de 65 segundos mais lento do que o cálculo com Emodels ( $p$.0001).
Os modelos de gesso tinham uma média para o comprimento do arco de $1,5( \pm 1,36 \mathrm{~mm})$

do que os modelos digitais Emodels pP .0001; intervalo).

Houve diferenças estatisticas significante entre os dois métodos ( $p$ <0.001). As medidas obtidas nos modelos digitais foram menores.

Houve diferenças significativas entre os modelos de gesso e os modelos digitais para contatos oclusais, relações oclusais e escores totais ( $p$ $<0,05)$. Nenhuma diferença significativa foi encontrada para alinhamento, cristas marginais, overjet e contatos interproximais.

Arco superior: erro médio de $0,14 \mathrm{~mm}$ Arco inferior: erro médio $0,18 \mathrm{~mm}$ A distância de $95 \%$ de todos os pontos correspondentes é entre 0,28 e 0,62 mm para a arcada superior e entre 0,35 e 0,64 $\mathrm{mm}$ para a arcada inferior.

Os resultados mostraram que $12,8 \%$ das características diagnósticas, $12 \%$ dos procedimentos mecânicos de tratamento e $6 \%$ dos planos de tratamento propostos mudaram após avaliação realizada em modelo de gesso $(p=.05)$. 
Tabela 5: Continuação

Ao comparar os instrumentos de medição, Observa-se que as medidas mesiodistais dos dentes 13, 14, 23, 33 e 35 foram

Sanches et al. (2013) estatisticamente significativamente maiores para medições realizadas pelo software 03d comparado com um paquímetro digital

$$
(p<0.05) \text {. }
$$

Houve diferença estatisticamente significante
$(p<0,05)$ entre as medições para a largura
dos dentes realizadas pelos dois métodos. As
medições nos modelos digitais apresentaram-
se menores do que as correspondentes
no modelo de gesso(média de $0,49 \mathrm{~mm})$,
a maior diferença foi encontrada para o
incisivo lateral esquerdo inferior $(0,38 \mathrm{~mm})$ e
overbite $(\mathrm{p}=0.0124)$.

Não houve diferença estatistica entre as

Sfondrini et al., (2017) medidas em modelo de gesso ou digital. No entanto, o tempo de processamento do modelo digital foi menor do que o método tradicional.

As avaliações de rotações nos modelos digitais apresentaram-se maiores. A técnica convencional mostrou menor variação Sjogren et al., (2010) intraexaminador para variáveis angulares do que o método de imagem 3D.

A maioria das diferenças médias no tamanho dos dentes não foram estatisticamente significantes $(p>0.0021=0.05)$

Stevens et al., (2006)

$(p>0.0021=0,05 / 24)$. A diferença das medidas das larguras dos dentes foi de $0,17 \mathrm{~mm}$ para os modelos de gesso e 0,22 para os modelos digitais Emodels.

Não houve diferenças estatisticas significativas foram observadas em relação às medidas

Tavares et al., (2017) realizadas no gesso ou modelos digitais ( $p>$ 0.05).

Não foram encontrados valores estatisticamente diferentes para a pontuação total do Índice de complexidade, resultado e necessidade (ICON) em modelos de gesso ou

Veenema et al., (2009) digitais. As pontuações do ICON realizadas em modelos baseados em computador parecem ser tão precisas e confiáveis quanto às pontuações do ICON em modelos de gesso.

Todos os valores obtidos a partir dos modelos digitais da Cécile 3 foram menores que os valores obtidos nos modelos de gesso $(p<0.05)$. 
Tabela 5: Continuação

As diferenças médias entre os modelos de gesso e digital em relação análise de Bolton anterior e global para o examinador 1 foram de $0,34 \mathrm{~mm}$ para ambos e para o

Watanabe-Kanno et al. (2010) examinador 2 foram de $0,28 \mathrm{~mm}$ e 0,21 $\mathrm{mm}$, respectivamente. Embora as medidas do modelo digital tenham sido inferiores ao gesso $(p<0.05)$, estas foram consideradas clinicamente insignificantes (diferenças $<0,1 \mathrm{~mm}$ ).

As diferenças das medições nos modelos de gesso variaram de 0,04 a $0,16 \mathrm{~mm}$ para os modelos digitais no DigiModels e de 0,24 a 0,07 $\mathrm{mm}$ para os modelos no Lava

Wiranto et al. (2013)

Scanner: média de 23 minutos

Moldels. Nenhuma medidanos modelos Lava foi significativamente diferente daqueles nos modelos de gesso. Das medições no DigiModels, alguns mostraram diferenças significativas $(p<0.05)$. No entanto, nenhuma diferença ultrapassou 0,2 $\mathrm{mm}$.

Yuan et al., (2013) 20 minutos

Diferença de $0.04 \mathrm{~mm}$, sem significância estatistica $(p=0.05)$

Não houve diferença significante entre os modelos de gesso e os modelos digitais, exceto por uma medida de menor largura

Zhang et al., (2017) intermediária $(P<0,005)$. A diferença média de superfície entre os dois modelos foi de

$$
0,10 \mathrm{~mm} \text {. }
$$

Houve correlação entre as medidas da largura dentária, largura intercanina e intermolar $(p=0.05)$.

\section{DISCUSSÃO}

A presente revisão sistemática centrou-se na avaliação da confiabilidade das medidas realizadas em modelos ortodônticos digitais comparados aos modelos de gesso convencionais. Foram incluídos estudos laboratoriais utilizando modelos ortodônticos digitais, onde os modelos de gesso funcionaram como grupo controle. Buscou-se assim, evidências na literatura sobre a confiabilidade de modelos ortodônticos digitais, visando a apresentação de dados calculados estatisticamente.

A importância desse estudo deve-se ao fato de avaliar as evidências a respeito da confiabilidade dos modelos digitais para serem utilizados na prática clínica dos ortodontistas, eliminando a necessidade de espaço para acomodação desses.

\section{Modelos digitais menos confiáveis}

Os AnatoModels apresentaram-se como os modelos menos acurados $(p<0.05)$ quando comparados com o grupo controle (GRUNHEID et al., 2014; LUU et al., 2014), demandando quase o dobro de tempo requerido para modelos de gesso quando da sua obtenção. Além disso, notou-se uma necessidade das medidas lineares e categóricas serem avaliadas com cuidado ao se utilizar desse processo de análise, no que se refere ao envolvimento de erro sistemático (LUU et al., 2014). Os modelos do sistema O3DM (versão 2.2) mostraram reprodutibilidade aceitável, porém esta foi menor para vários ângulos, com limite de concordância pobre para variáveis angulares e lineares (SJOGREN et al., 2010).

\section{Modelos digitais igualmente confiáveis}

As medidas nos modelos de gesso e RepRap3D 
foram equivalentes. A análise da precisão foi feita atavés dos valores de desvio padrão, os quais não diferiram significativamente da soma do desvio padrão dos modelos de gesso, sendo menor que 0,5 $\mathrm{mm}(\mathrm{p}=0.38)$. Entretanto, os modelos de RepRap3D apresentaram a vantagem de serem mais rápidos $\mathrm{e}$ terem menor custo (KASPAROVA et al., 2013). Além desses, os modelos Orthocad também apresentaram mesma acurácia e confiabilidade que os modelos de gesso, quanto a análise de espaço, sendo as diferenças clinicamente insignificantes ( $p<0.05$ ) (LEIFERT et al., 2009). Contudo foi demonstrado que apesar de ser uma alternativa(SANTORO et al., 2003; COSTALOS et al., 2005) aos modelos de gesso o software Orthocad ABO OGS em sua versão digital não substitui o sistema manual, pois não fornece o mesmo escore e são inacurados quando em oclusão, interferindo nas medidas de overjet e contatos oclusais(HILDEBRAND et al., 2008). Os modelos Emodel (versão 6.0, GeoDigm) apresentaram-se com acurácia similar aos modelos de gesso e igualmente confiável aos mesmos, porém com tempo útil de mensuração mais rápido (MULLEN et al., 2007). As medidas da largura dos dentes foram no geral similares entre modelos de gesso e DigitiModels e Lava models, não excedendo $0,2 \mathrm{~mm}$ e sendo tão confiáveis quanto os mesmos (WIRANTO et al., 2013). As mensurações realizadas pelo software Ortho Analyzer apresentaram diferenças entre os modelos digitas e de gesso, no entanto, essa diferença foi considerada clinicamente aceitavel(CZARNOTA et al., 2016; CAMARDELLA et al., 2017; SFONDRINI et al., 2018). Outros modelos digitais mensurados nos softwares Cloud (GREWAL et al., 2017) e Onyx Ceph3 (JIMENEZ-GAYOSSO et al., 2018) apresentaram boa confiabilidade, apesar de apresentarem diferenças em alguns parametros, mas com aceitação clinica.

\section{Modelos digitais mais confiáveis}

As medidas realizadas em modelos SureSmile (GRUNHEID et al., 2014) e alguns Emodels (STEVENS et al., 2006; GRUNHEID et al., 2014) demostram-se mais confiáveis. Grunheid et al. (2014) demonstraram que modelos digitais podem ser tão acurados, mais reprodutíveis e significativamente mais rápidos do que mensurações em modelos de gesso. As medidas mais precisas foram atribuídas aos SureSmile, seguida pelos Emodels. No que tange a confiabilidade dos modelos Emodels, utilizando a média das medidas repetidas, a diferença média intraexaminador foi levemente maior na forma digital, enquanto a média interexaminador foi similar. A reprodutibilidade intra e interexaminador no geral foram maiores para os modelos digitais (STEVENS et al., 2006).

Assim como, os modelos digitais da Ivori Analyze foram considerados confianveis, uma vez que houve menor variação entre as medições repetidas, no entanto, as medidas da largura dos dentes na Analise Bolton foram maiores que os do modelo de gesso, apresentando diferença de $0,5 \mathrm{~mm}$ entre os modelos. (KORETSI et al., 2018).

Apesar de notável a evolução da tecnologia digital, ainda é observada limitações quanto a precisão na análise de alguns desses modelos. Nessas condições, conforme apresentado pelos estudos prévios de Grunheid et al 2014 e Luu et al 2014, os modelos digitais da Anatomodels preencheram os caracteres de menor acurácia na reprodução das mensurações realizadas em modelos ortodônticos. Sendo assim, caberia salientar a importância do diagnóstico complementar, que poderia nesses casos, ser um coadjuvante a análise dos convencionais modelos de gesso, melhorando a qualidade do planejamento, já que, por si só, não forneceriam informações suficientemente adequadas para a prática clínica. Por outro lado, foi demonstrado que já existem modelos digitais, considerados altamente precisos, sendo tão confiáveis quanto em modelos de gesso, representando assim uma evidência positiva para a substituição destes, ao se considerar os benefícios inerentes.

\section{CONCLUSÃO}

Pode-se inferir com a realização dessa revisão sistemática que:

- As mensurações realizadas em modelos digitais podem ser tão bem acuradas, reprodutíveis e confiáveis quanto o método convencional utilizando modelos de gesso.

\section{REFERÊNCIAS}

ABIZADEH, N. et al. Digital versus plaster study models: how accurate and reproducible are they? Journal of Orthodontics, $v$. 39, n. 3, p. 151-9, sep. 2012.

ASQUITH, J. A.; MCINTYRE, G. T. Dental arch relationships on three-dimensional digital study models and conventional plaster study models for patients with unilateral cleft lip and palate. The Cleft Palate-Craniofacial Journal, v. 49, n. 5, p. 530-4, sep. 2012.

BELL, A.; AYOUB, A. F.; SIEBERT, P. Assessment of the accuracy of a three-dimensional imaging system for archiving dental study models. Journal of Orthodontics, v. 30, n. 3, p. 219-23, sep. 2003.

BOOTVONG, K. et al. Virtual model analysis as an alternative approach to plaster model analysis: reliability and validity. The European Journal of Orthodontics, v. 32, n. 5, p. 589-95, oct. 2010.

CAMARDELLA, L. T.; BREUNING, H.; DE VASCONCELLOS VILELLA, $O$. Accuracy and reproducibility of measurements on plaster models and digital models created using an intraoral scanner. Journal of Orofacial Orthopedics, v. 78, n. 3, p. 211-220, may. 2017. 
COSTALOS, P. A. et al. Evaluation of the accuracy of digital model analysis for the American Board of Orthodontics objective grading system for dental casts. American Journal of Orthodontics and Dentofacial Orthopedics, v. 128, n. 5, p. 624-629, nov. 2005.

CZARNOTA, J.; HEY, J.; FUHRMANN, R. Measurements using orthodontic analysis software on digital models obtained by $3 \mathrm{D}$ scans of plaster casts : Intrarater reliability and validity. Journal of Orofacial Orthopedics, v. 77, n. 1, p. 22-30, jan. 2016.

DALSTRA, M.; MELSEN, B. From alginate impressions to digital virtual models: accuracy and reproducibility. Journal of Orthodontics, v. 36, n. 1, p. 36-41; discussion 14, mar 2009.

EL-ZANATY, H. M. et al. Three-dimensional dental measurements: An alternative to plaster models. American Journal of Orthodontics and Dentofacial Orthopedics, v. 137, n. 2, p. 259-65, feb. 2010.

FLEMING, P. S.; MARINHO, V.; JOHAL, A. Orthodontic measurements on digital study models compared with plaster models: a systematic review. Orthod Craniofac Res, v. 14, n. 1, p. 1-16, feb. 2011.

$\mathrm{FU}, \mathrm{X}$. et al. The application of multi-baseline digital close-range photogrammetry in three-dimensional imaging and measurement of dental casts. PLoS One, v. 12, n. 6, p. e0178858, 2017.

GREWAL, B. et al. Royal London space analysis: plaster versus digital model assessment. The European Journal of Orthodontics, $v$. 39, n. 3, p. 320-325, jun. 12017.

GRUNHEID, T. et al. Accuracy, reproducibility, and time efficiency of dental measurements using different technologies. American Journal of Orthodontics and Dentofacial Orthopedics, v. 145, n. 2, p. 157-64, feb. 2014.

HAZEVELD, A.; HUDDLESTON SLATER, J. J.; REN, Y. Accuracy and reproducibility of dental replica models reconstructed by different rapid prototyping techniques. American Journal of Orthodontics and Dentofacial Orthopedics, v. 145, n. 1, p. 108-15, jan. 2014. HILDEBRAND, J. C. et al. Evaluation of a software program for applying the American Board of Orthodontics objective grading system to digital casts. American Journal of Orthodontics and Dentofacial Orthopedics, v. 133, n. 2, p. 283-9, feb. 2008.

IM, J. et al. Comparison of virtual and manual tooth setups with digital and plaster models in extraction cases. American Journal of Orthodontics and Dentofacial Orthopedics, v. 145, n. 4, p. 434-42, apr. 2014.

JIMENEZ-GAYOSSO, S. I. et al. Difference between manual and digital measurements of dental arches of orthodontic patients. Medicine (Baltimore), v. 97, n. 22, p. e10887, jun. 2018.

JOFFE, L. OrthoCAD: digital models for a digital era. Journal of Orthodontics, v. 31, n. 4, p. 344-7, dec. 2004.
KANG, S. H. et al. Early orthognathic surgery with three-dimensional image simulation during presurgical orthodontics in adults. Journal of Craniofacial Surgery, v. 22, n. 2, p. 473-81, mar. 2011.

KASPAROVA, M. et al. Possibility of reconstruction of dental plaster cast from 3D digital study models. BioMedical Engineering OnLine, v. 12, p. 49, may. 2013.

KEATING, A. P. et al. A comparison of plaster, digital and reconstructed study model accuracy. Journal of Orthodontics, $v$. 35, n. 3, p. 191-201; discussion 175, sep. 2008.

KIM, J.; HEO, G.; LAGRAVERE, M. O. Accuracy of laser-scanned models compared to plaster models and cone-beam computed tomography. The Angle orthodontist, v. 84, n. 3, p. 443-50, may. 2014.

KIM, J. H. et al. Accuracy and precision of polyurethane dental arch models fabricated using a three-dimensional subtractive rapid prototyping method with an intraoral scanning technique. The Korean Journal of Orthodontics, v. 44, n. 2, p. 69-76, mar. 2014. (a)

KIM, S. Y. et al. Comparison of the accuracy of digitally fabricated polyurethane model and conventional gypsum model. The Journal of Advanced Prosthodontics, v. 6, n. 1, p. 1-7, feb. 2014.

KORETSI, V. et al. Intra-observer reliability and agreement of manual and digital orthodontic model analysis. The European Journal of Orthodontics, v. 40, n. 1, p. 52-57, jan. 2018.

LEIFERT, M. F. et al. Comparison of space analysis evaluations with digital models and plaster dental casts. American Journal of Orthodontics and Dentofacial Orthopedics, v. 136, n. 1, p. 16 e1-4; discussion 16, jul. 2009.

LUU, N. S. et al. The validity, reliability, and time requirement of study model analysis using cone-beam computed tomographygenerated virtual study models. Orthodontics \& Craniofacial Research, v. 17, n. 1, p. 14-26, feb. 2014.

$M A H, J$. The evolution of digital study models. Journal of Clinical Orthodontics v. 41, n. 9, p. 557-61; quiz 424, sep. 2007.

MANGIACAPRA, R. et al. Traditional plaster casts and dental digital models: intra-examiner reliability of measurements. Progress in Orthodontics, v. 10, n. 2, p. 48-53, 2009.

MULLEN, S. R. et al. Accuracy of space analysis with emodels and plaster models. American Journal of Orthodontics and Dentofacial Orthopedics, v. 132, n. 3, p. 346-52, sep. 2007.

MURUGESAN, $K$. et al. Comparative evaluation of dimension and surface detail accuracy of models produced by three different rapid prototype techniques. The Journal of the Indian Prosthodontic Society, v. 12, n. 1, p. 16-20, mar. 2012. 
NALCACI, R.; TOPCUOGLU, T.; OZTURK, F. Comparison of Bolton analysis and tooth size measurements obtained using conventional and three-dimensional orthodontic models. European Journal of Dentistry, v. 7, n. Suppl 1, p. S66-70, sep. 2013.

OKUNAMI, T. R. et al. Assessing the American Board of Orthodontics objective grading system: digital vs plaster dental casts. American Journal of Orthodontics and Dentofacial Orthopedics, v. 131, n. 1, p. 51-6, jan 2007.

RANGEL, F. A. et al. Accuracy and reliability of a novel method for fusion of digital dental casts and Cone Beam Computed Tomography scans. PLoS One, v. 8, n. 3, p. e59130, 2013. RHEUDE, B. et al. An evaluation of the use of digital study models in orthodontic diagnosis and treatment planning. The Angle orthodontist, v. 75, n. 3, p. 300-4, may. 2005.

SANCHES, J. O. et al. Comparison of space analysis performed on plaster vs. digital dental casts applying Tanaka and Johnston's equation. Dental Press Journal of Orthodontics, v. 18, n. 1 , p. 128-33, jan./feb. 2013.

SANTORO, M. et al. Comparison of measurements made on digital and plaster models. American Journal of Orthodontics and Dentofacial Orthopedics, v. 124, n. 1, p. 101-5, jul. 2003.

SFONDRINI, M. F. et al. Computerized Casts for Orthodontic Purpose Using Powder-Free Intraoral Scanners: Accuracy, Execution Time, and Patient Feedback. BioMed Research International, v. 2018, p. 1-8.

SJOGREN, A. P.; LINDGReN, J. E.; HUGGARE, J. A. Orthodontic study cast analysis--reproducibility of recordings and agreement between conventional and 3D virtual measurements. Journal of Digital Imaging, v. 23, n. 4, p. 482-92, aug. 2010.

STEVENS, D. R. et al. Validity, reliability, and reproducibility of plaster vs digital study models: comparison of peer assessment rating and Bolton analysis and their constituent measurements. American Journal of Orthodontics and Dentofacial Orthopedics, v. 129, n. 6, p. 794-803, jun. 2006.

TAVARES, A.; BRAGA, E.; ARAUJO, T. M. Digital models: How can dental arch form be verified chairside? Dental Press Journal of Orthodontics, v. 22, n. 6, p. 68-73, nov./dec. 2017.

VEENEMA, A. C. et al. Index of Complexity, Outcome and Need scored on plaster and digital models. The European Journal of Orthodontics, v. 31, n. 3, p. 281-6, jun. 2009.

WATANABE-KANNO, G. A. et al. Determination of tooth-size discrepancy and Bolton ratios using Bibliocast Cecile3 digital models. International Orthodontics, v. 8, n. 3, p. 215-26, sep. 2010.
WATANABE-KANNO, G. A. et al. Reproducibility, reliability and validity of measurements obtained from Cecile 3 digital models.

Brazilian Oral Research, v. 23, n. 3, p. 288-95, jul./sep. 2009.

WIRANTO, M. G. et al. Validity, reliability, and reproducibility of linear measurements on digital models obtained from intraoral and cone-beam computed tomography scans of alginate impressions. American Journal of Orthodontics and Dentofacial Orthopedics, v. 143, n. 1, p. 140-7, jan. 2013.

YUAN, F. S. et al. Accuracy evaluation of a new three-dimensional reproduction method of edentulous dental casts, and wax occlusion rims with jaw relation. International Journal of Oral Science, v. 5, n. 3, p. 155-61, sep. 2013.

ZHANG, F.; SUH, K. J.; LEE, K. M. Validity of Intraoral Scans Compared with Plaster Models: An In-Vivo Comparison of Dental Measurements and 3D Surface Analysis. PLoS One, v. 11, n. 6, p. e0157713, 2016.

ZILBERMAN, O.; HUGGARE, J. A.; PARIKAKIS, K. A. Evaluation of the validity of tooth size and arch width measurements using conventional and three-dimensional virtual orthodontic models. The Angle orthodontist, v. 73, n. 3, p. 301-6, jun. 2003 